\title{
The Evolving Role for Autologous Adipose Tissue (Fat) in Treating Hair Loss
}

\author{
Gorana Kuka Epstein, MD I Belgrade, Serbia I gorana.kuka@me.com I Jeffrey S. Epstein, MD I Miami, Florida, USA
}

Regenerative therapy is increasingly being recognized for its potential role in the treatment of a variety of types of hair loss. The most accessible such procedure, and therefore of greatest familiarity to hair restoration surgeons, is platelet rich plasma (PRP) therapy. Not only is it easy to obtain, there are no restrictions on the use of PRP in the United States or, to our knowledge, in any other country. PRP clearly has some efficacy, but it is unclear how much, partly due to a lack of uniform standards and how it is administered, and partly due to a lack of quality studies. PRP proponents claim several benefits including the following: 1) a reduction in shock loss and expedited healing when performed in conjunction with a hair transplant, 2) a reduction in hair shedding and reversal of hair miniaturization, and 3) a slowing of the progression of male and female hair loss when performed as a stand-alone treatment.

Regenerative cells, a more cutting-edge therapy, are currently used by a variety of specialties including cardiology, neurology, dermatology, and immunology. The recently completed first FDA-approved study on adipose-derived regenerative cells (ADRC) for the treatment of androgenetic alopecia demonstrated potential benefits in early to mid male pattern hair loss, a study in which the authors were co-investigators (publication in preparation, ClinicalTrials.gov identifier: NCT0250352). Approval of this therapy for treating hair loss is still pending in the United States, but it is approved for use in Europe. In this article, we describe an alternative therapy-autologous fat transfer with PRP — that may harness some of the benefits of while minimizing the downsides of PRP and stem cell therapy.

\section{AUTOLOGOUS FAT TRANSFER WITH PRP}

Since 2012, we have been investigating the efficacy of autologous adipose tissue (fat) for the treatment of a variety of hair loss conditions. Our earliest application was for the pretreatment of scar tissue prior to a hair transplant, theoretically taking advantage of some of the characteristics of fat to optimize the regrowth of transplanted hairs (Figure 1). These characteristics include promotion of angiogenesis, thus improving the vascularity, and the softening and thickening (when indicated) of scar tissue. ${ }^{1}$

Enthusiastic about our positive results for this indication (Figure 1), we began to study this fat therapy for cases of cicatricial alopecias, then more recently extending its application to the treatment of pattern hair loss in women and men (Figures 2 through 6). We have similarly observed potential benefits that are presented below.

Adipose tissue has a variety of characteristics that make it potentially effective in treating hair loss. First, it is the largest repository of stem cells in the adult body, and the stem cells can be obtained relatively easily through liposuction as

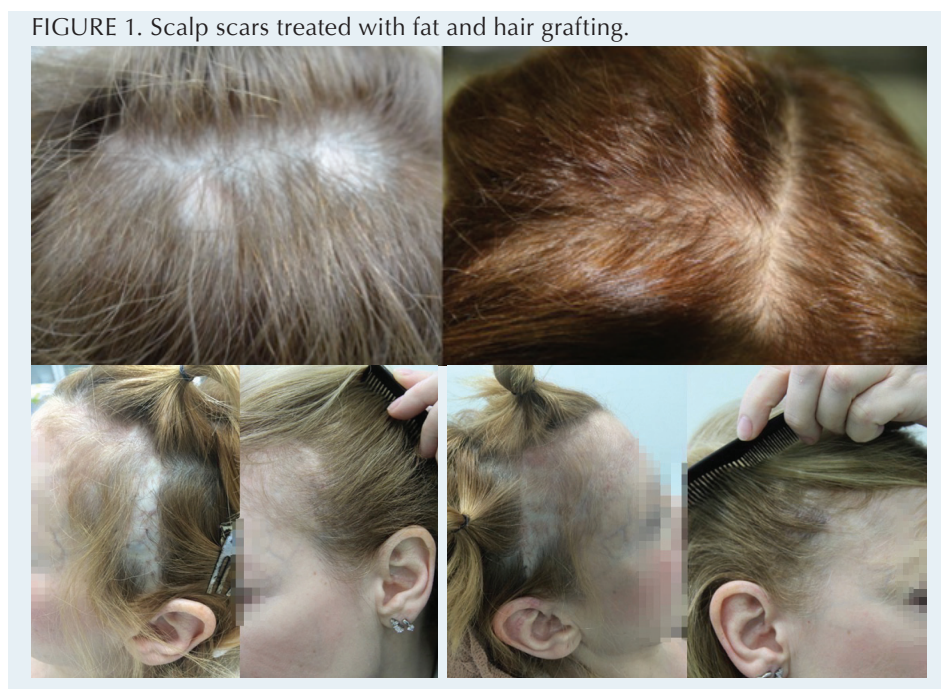

opposed to the challenges of harvesting stem cells from bone marrow. ${ }^{2}$ Stem cells have shown at least some efficacy for the treatment of pattern baldness. Second, the tissue possesses anti-androgen activity, androgens being an element in the progression of male pattern hair loss (MPHL) and possibly female pattern hair loss (FPHL). ${ }^{3}$ Third, adipose tissue possesses anti-inflammatory properties. Inflammation is associated with, if not a contributing factor to, pattern baldness, with mild perifollicular fibrosis and infiltrates found in some patients with androgenetic alopecia. ${ }^{4}$ Adipose-derived stem cells may prevent further inflammation and possible damage to hair follicles through enhancement of anti-oxidative and anti-inflammatory mechanisms. Fourth, as described above, adipose tissue induces neovascularization, and can potentially thicken the subcutaneous layer that typically is associated with thinning in androgenetic alopecia. ${ }^{5}$

Given these characteristics, we have developed a protocol, which we are continually modifying and fine-tuning, for autologous fat transfer with PRP (AFTP). At the time of this writing, we have used AFTP on close to 100 patients.

\section{Technique}

Pre-procedure, the potential benefits, risks, and limitations are reviewed, emphasizing that, similar to PRP, this is a therapy with inconsistent results, although it does seem to be more effective. Pre-op CBC and PT/PTT levels are obtained, and patients are instructed to avoid all aspirin for 10 days. The area(s) of the scalp to be treated are determined, and the desired amount of purified adipose tissue needed is then determined as well. Typical volumes of adipose tissue range from 10-20cc for treating small areas, to 40-60cc for treating the entire top of the scalp extending from the frontal hairline to crown region and laterally to the upper sides. The donor area for the adipose tissue is most commonly the central lower 
FIGURE 2. 57-year-old woman with female pattern hair loss treated with a single procedure resulted in less shedding and narrowing of the part line.

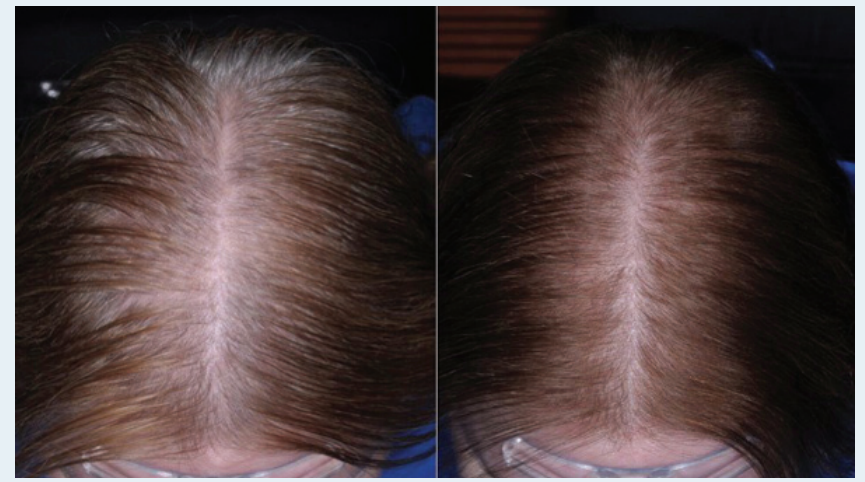

FIGURE 3. 27-year-old male AGA patient treated with a single procedure resulted in thickening of hair.

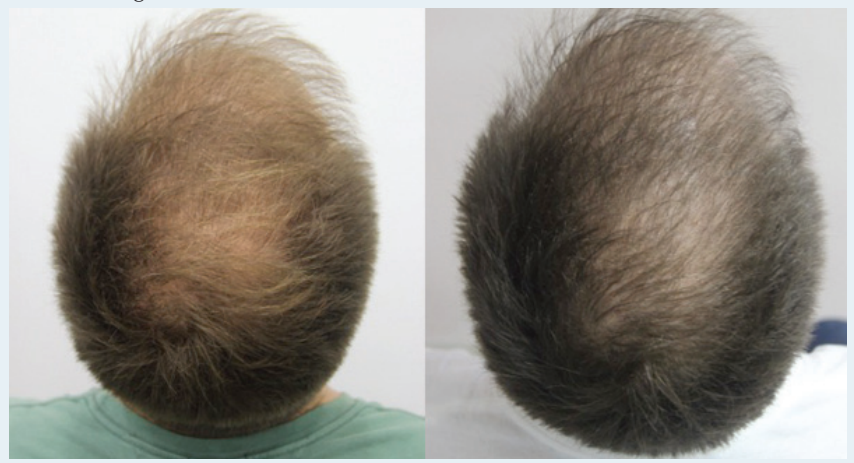

FIGURE 4. 52-year-old male AGA patient treated with a single procedure resulted in thickening of hair.

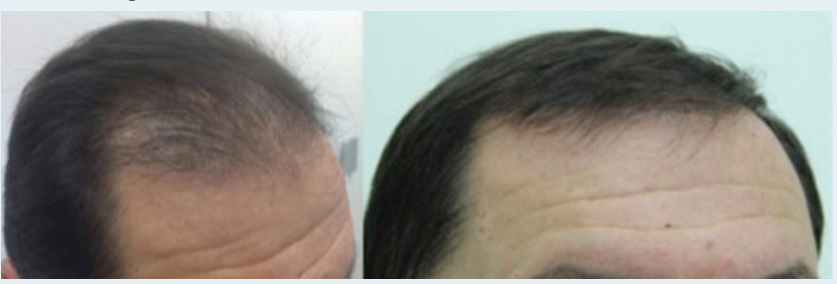

FIGURE 5. 42-year-old female with frontal fibrosing alopecia treated with adipose tissue grafting only. Biopsy was done prior to and 3 months after the procedure and confirmed that folliculo-sebaceous architectural disruption was less pronounced. Overall, treated area seemed smoother.

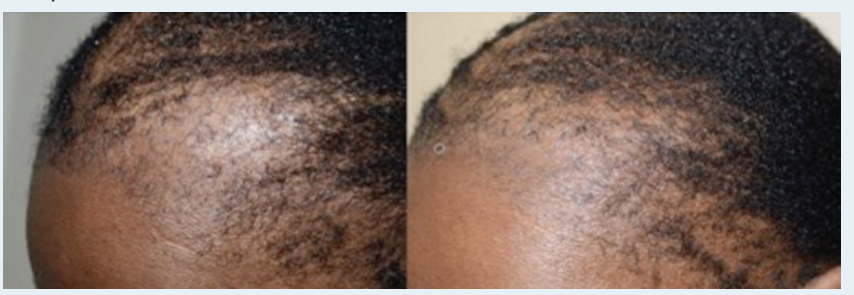

FIGURE 6. 38-year-old female with alopecia universalis had the backside of her head treated with adipose tissue grafting with PRP. Regrowth was noticed 6 months after the procedure.

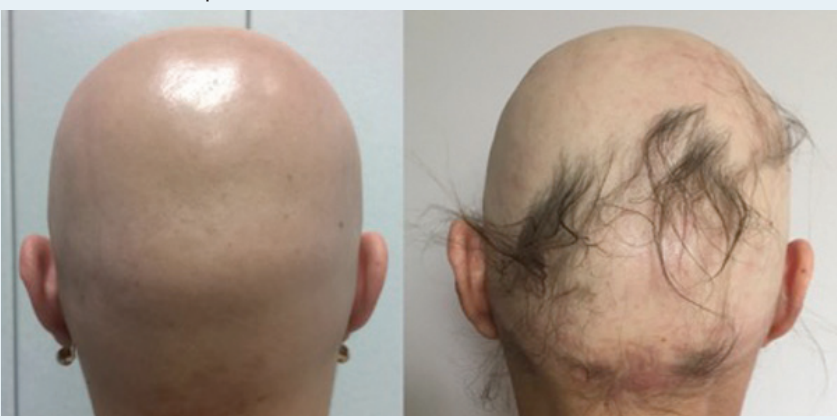

abdomen both above and/or below the umbilicus, followed by the upper hips, then least commonly the lateral thighs.

The procedure is usually performed under local anesthesia with light oral sedation, although twilight sedation can be offered. Under sterile conditions, the selected area(s) for liposuction is (are) infiltrated with tumescence containing 160cc of saline, 20cc of lidocaine 2\%, 20cc of Marcaine, and 1cc of 1:1000 epinephrine. Waiting 15 minutes to allow the tumescence to become effective, a $3 \mathrm{~mm}$-diameter, $15 \mathrm{~cm}$-long 3 -hole distal-opening blunt-tipped cannula (Tulip ${ }^{\circledR}$ ) attached to a 10cc syringe is used to harvest the adipose tissue under negative pressure. The fat is then filtrated by the Puregraft ${ }^{\circledR}$, a closed membrane filtration system that removes tumescent fluid, oil, blood cells, and debris, leaving purified adipose tissue. We find this system superior to other fat processing methods due to its high percentage $(97 \%)$ of contaminant removal, low amount of tissue trauma, and ease of use compared to other methods of fat graft preparation (centrifuge, gravity separation, etc.). ${ }^{6}$

Venous blood is then obtained and processed using the RegenLab ${ }^{\circledR}$ system to obtain 8-10cc of PRP that is added to the adipose tissue with the typical ratio of 1:4 in favor of the fat. This adipose tissue with PRP is then placed into multiple 1cc Luer-lock syringes and injected into the subcutaneous layer of the affected portion(s) of the anesthetized scalp through a 21-gauge blunt cannula. The pattern of injection is of multiple tunnels into the affected areas, in different directions. This injecting is done slowly $(0.5-1 \mathrm{cc} /$ second) as studies have demonstrated a 38\% improvement in adipose tissue graft survival with slow versus fast (3-5cc/second) injections. ${ }^{7}$ (See Figure 7.) Antibiotic ointment is applied to the 18G puncture sites into which the blunt cannulas were introduced, while the entry sites for the liposuction cannulas are sutured, then a pressure dressing applied for 48 hours to the area. Patients are typically presentable the very next day with little to no scalp swelling, and are able to resume regular activities after three days.

\section{DISCUSSION}

Over the past 10 years, interest and acceptance of autologous adipose tissue grafting, mesenchymal stem cells (MSCs), stromal vascular fraction, and platelet rich plasma therapy have increased in many fields of medicine. Individualized or combined treatment has been effective in various medical conditions. Since hair loss remains a condition in which there is no universal definitive therapy that works in every patient, there is a potential role for regenerative therapy in treating different types of alopecias. Androgenetic alopecia (AGA) remains the main cause of hair loss both in men and women. ${ }^{8,9}$ The two medications currently approved by the FDA for AGA are finasteride (for men only) and minoxidil, however, both have potential side-effects ${ }^{10,11}$ and are ineffective in as many as $30-50 \%$ of patients. In addition, each needs to be used on a daily basis. The main roles of mesenchymal stem cells (MSCs) are to maintain the stem cell niche, facilitate recovery after injury, and ensure homeostasis of organs and tissues. ${ }^{12}$ Adipose tissue remains the most accessible source of MSCs besides bone marrow where they were first identified. Adipose-derived stem cells (ADSCs) can differentiate into mesenchymal lineage cells but also secrete various cytokines and growth factors that have 
FIGURE 7. $A$ : Mini-liposuction of adipose tissue; $B$ : purifying adipose tissue by Puregraft; $C$ : mixing PRP with adipose tissue; $D$ : injection of autologous adipose tissue enriched with PRP
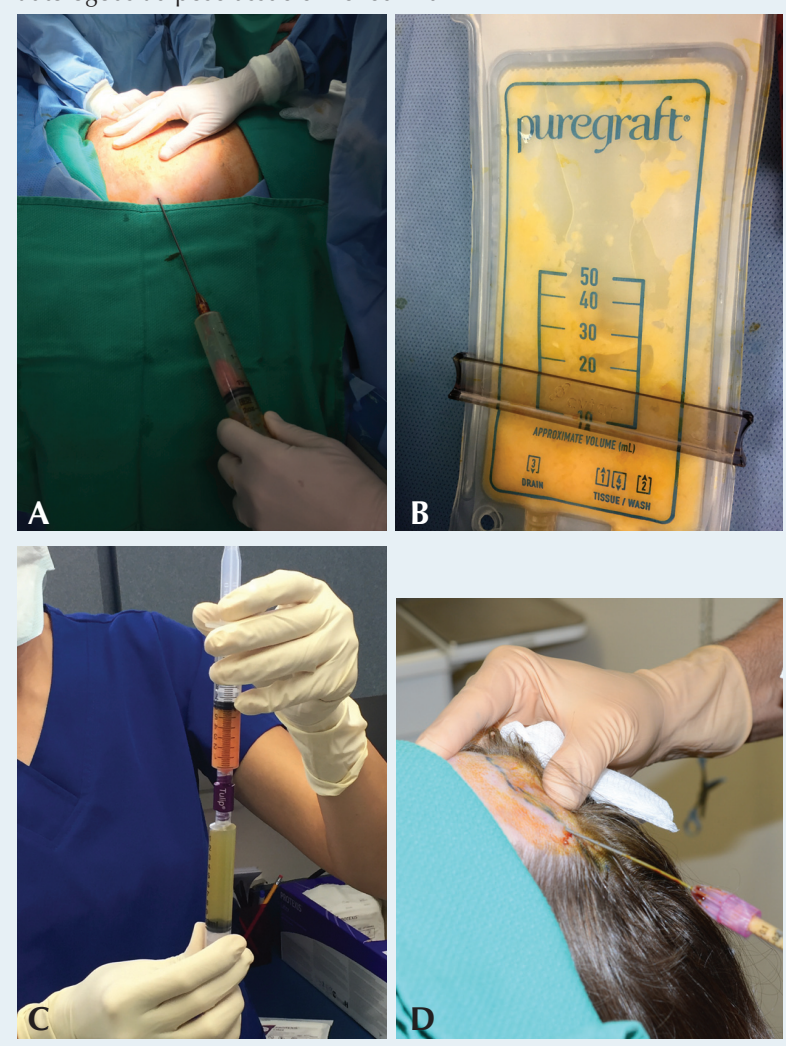

paracrine effects on surrounding cells: vascular endothelial growth factor (VEGF), hepatocyte growth factor (HGF), insulin-like growth factor (IGF), platelet-derived growth factors (PDGF), and others. ${ }^{13,14}$ These factors seem to play an important role in neovascularization, which is important in treating various hair loss conditions.

Festa et al. report that adipocyte lineage cells support the follicular stem cell niche and help drive the complex hair growth. ${ }^{15}$ This introduces primary evidence and supports the hypothesis that the fat depots in the scalp may play a role in hair loss and hair growth. Apart from androgenetic alopecia, the decision to treat frontal fibrosing alopecia (FFA) with autologous fat injection stems from a very successful trial that was done for treating patients with scleroderma. ${ }^{16}$ Since these two conditions are of a similar pathology (skin fibrosis) and much improvement was seen in the scleroderma study, it is our hope and early observation that fat will be beneficial for FFA patients and can serve as pretreatment for hair transplant procedures once the degree of fibrosis is somewhat reduced.

The addition of PRP has benefits not just in growth factors released from thrombocytes, but also in promoting the proliferation of stem cells and fibroblasts. The addition of 20\% PRP to adipose tissue ensures better fat graft survival and somewhat easier application due to liquefaction effect of PRP.

The procedure has been well tolerated by patients, and only minor side effects have been noted, such as mild swelling and erythema. Abdominal lipo-aspiration entry sites healed at one week, and the points for fat injection healed as soon as the next day. It takes two to three months to see some results of the treatment although patients reported satisfaction even sooner.

\section{Final thoughts}

The early results we have achieved using fat for a variety of hair loss conditions makes us eager to continue this investigative work. Initially applied to enhance the outcomes from hair transplants into scar tissue, we have extended its application as we have found it to be potentially effective as a stand-alone intervention for treating earlier stages of male and female pattern hair loss. We also are optimistic that it can play a role in the difficult management of cicatricial alopecias.

Adipose tissue possesses many special characteristics that theoretically should make it effective in treating pattern hair loss. Our results to date in this application support our continued investigative work on this therapy that has several advantages over stem cells. These advantages include a considerably lower cost to obtain, a much lower quantity of adipose tissue liposuction needed that is potentially within the skills of most hair restoration surgeons or learnable through tutoring, and the absence of the restrictions that are imposed in the United States on the use of stem cells.

\section{References}

1. Clinger, M., et al. Autologous fat graft in scar treatment. / Craniofac Surg. 2013; 24:1610-1615.

2. Zuk, P.A., et al. Human adipose tissue is a source of multipotent stem cells. Molecular Biology of the Cell. 2002; 13(2):4279-4295.

3. O'Reilly, M.W., et al. Understanding androgen action in adipose tissue. J of Steroid Biochem Mol Biol. 2014; 143:277-284.

4. Whiting, D.A. Scalp biopsy as a diagnostic and prognostic tool in androgenetic alopecia. Dermatol Ther. 1998; 8:24-33.

5. Hori, H., et al. The thickness of human scalp: normal and bald. J Inv Derm. 1972; 6:396-399.

6. Zhu, M., et al. Comparison of three different fat graft preparation methods: gravity separation, centrifugation, and simultaneous washing with filtration in a closed system. Plast Reconstr Surg. 2013; 131(4):883-870.

7. Lee, J.H., et al. The effect of pressure and shear on autologous fat grafting. Plast Recon Surg. 2013; 131(5):1125-1136.

8. Otberg, N., A.M. Finner, and J. Shapiro. Androgenetic alopecia. Endocrinol Metab Clin North Am. 2007; 36(2):379-398.

9. Dinh, Q.Q., and R. Sinclair. Female pattern hair loss: current treatment concepts. Clinical Interventions in Aging. 2007; 2(2):189-199.

10. Ross, E.K., and J. Shapiro. Management of hair loss. Dermatol Clin. 2005; 23(2):227-243.

11. Mysore, V. Finasteride and sexual side effects. Indian Dermatol Online J. 2012; 23(2):227-243.

12. Shin, H., et al. Up-to-date clinical trials of hair regeneration using conditioned media of adipose-derived stem cells in male and female pattern hair loss. Curr Stem Cell Res Ther. 2017(May 4).

13. Rehman, J., et al. Secretion of angiogenic and antiapoptotic factors by human adipose stomal cells. Circulation. 2004; 109:1292-1298.

14. Kinniard, T., et al. Narrow-derived stromal cells express gene encoding a broad spectrum of arteriogenic cytokines and promote in vitro and in vivo arteriogenesis through paracrine mechanisms. Circ Res. 2004; 94:678-685.

15. Festa, E., et al. Adipocite lineage cells contribute to the skin stem cell niche to drive hair cycling. Cell. 2011; 146(5):762-771.

16. Magalon, G., et al. Regenerative approach to scleroderma with fat grafting. Clin Plast Surg. 2015; 42:353-364. 